\title{
Enhancing Performance of a GaAs/AlGaAs/GaAs Nanowire Photodetector Based on the Two-Dimensional Electron-Hole Tube Structure
}

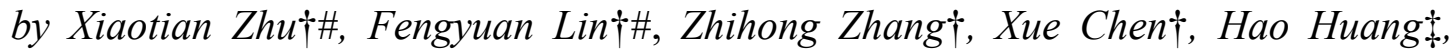

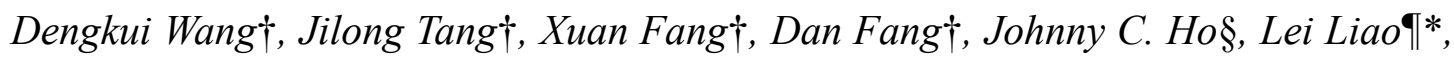
Zhipeng Wei†*

$\uparrow$ State Key Laboratory of High Power Semiconductor Lasers, Changchun University of Science and Technology, Changchun 130022, China

$\$$ Department of Physics and Key Laboratory of Artificial Micro-and Nano-structures of Ministry of Education, Wuhan University, Wuhan 430072, China

$\S$ Department of Materials Science and Engineering, City University of Hong Kong, Tat Chee Avenue, Kowloon, Hong Kong SAR 999077, China

IState Key Laboratory for Chemo/Biosensing and Chemometrics, School of Physics and Electronics, Hunan University, Changsha 410082, China 


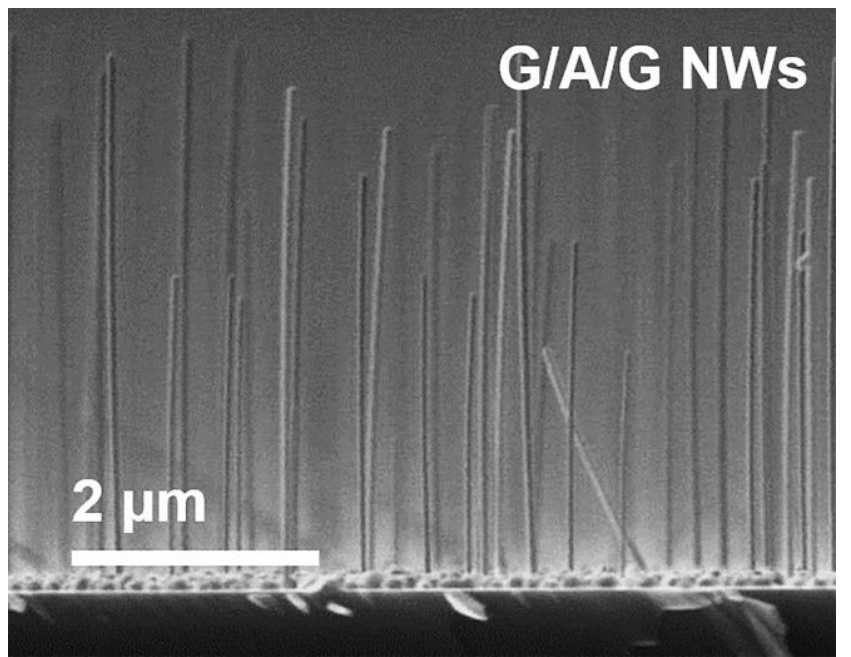

Figure S1. SEM image of typical G/A/G NW arrays.

The SEM image of these grown G/A/G NW arrays is shown in Figure S1. It is explicit that the NW arrays have a good verticality. The average length and diameter of the obtained G/A/G NWs are about $4 \mu \mathrm{m}$ and $200 \mathrm{~nm}$, respectively. 

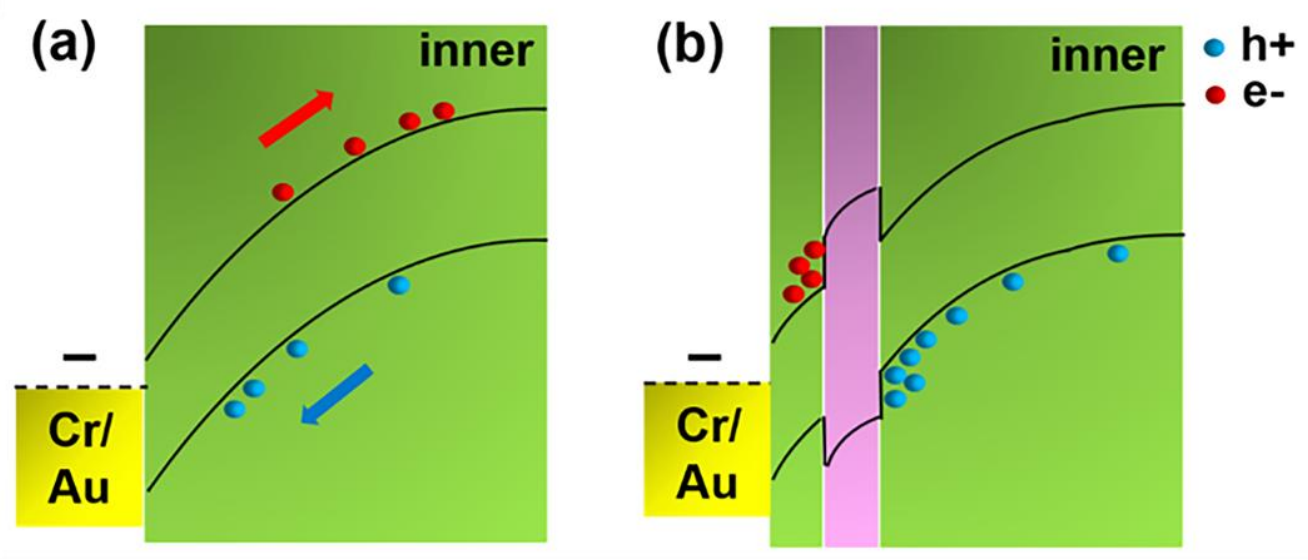

Figure S2. (a) Schematic illustration of the energy band diagram of GaAs NW at $-5 \mathrm{~V}$ bias. (b) Schematic illustration of the energy band diagram of G/A/G NW at $-5 \mathrm{~V}$ bias.

Figure S2 illustrates the band structure of both GaAs and G/A/G NWs contacting with metal electrodes under a bias of $-5 \mathrm{~V}$. Because of the Schottky barrier formed in the contact region between p-type GaAs and metal, the energy band would bend downward. Under a bias of $-5 \mathrm{~V}$, this downward bending would become larger in the magnitude as electrons and holes are transmitted to opposite sides of the electrodes as shown in Figure S2a. Since the intrinsic conduction and valence band offsets of GaAs/ $/ \mathrm{Al}_{0.4} \mathrm{Ga}_{0.6} \mathrm{As}$ are 0.316 and $0.184 \mathrm{eV}$, respectively, once the externally generated photoelectrons are transported to the inner NW layer, they are blocked and aggregated by the barrier formed by outer GaAs layer and AlGaAs layer. This way, a 2DET would be established at the outer $\mathrm{AlGaAs} / \mathrm{GaAs}$ interface, inducing photogenerated electrons to propagate axially and suppressing the radial carrier diffusion. Meanwhile, the photogenerated holes produced by internal GaAs layer are blocked by AlGaAs barrier layer, and would move to metal electrode under the electric field, forming a 2DHT at the inner AlGaAs/GaAs interface. The 2DHT would inhibit the radial carrier diffusion 
and reduce the recombination of carriers in the inner NW layer. In this manner, the photogenerated electron and hole pairs created in both inner and outer NW layers can be effectively separated by the 2DEHT as illustrated in Figure S2b. 


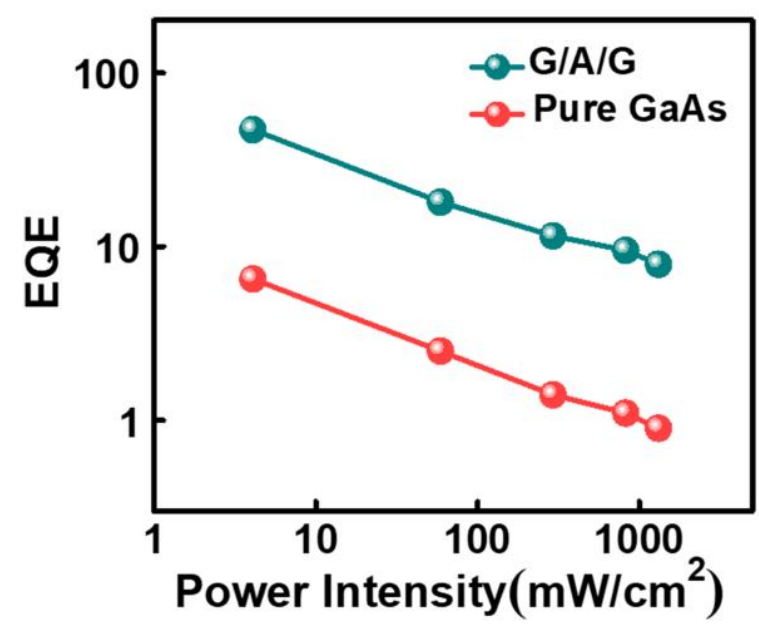

Figure S3. External quantum efficiency of the photodetectors made of GaAs and G/A/G NWs with illumination (808 nm) under an applied bias of $5 \mathrm{~V}$.

Moreover, we as well calculate the external quantum efficiency $(E Q E)$ of these two NW photodetectors in Figure S3. $E Q E$ is an important performance parameter of photodetectors. It is defined as the ratio of the number of carriers collected to the number of all incident photons. ${ }^{1}$ Generally, EQE can be calculated as follows:

$$
E Q E=\frac{I_{\text {light }} / e}{P A / h v}
$$

where $h$ is Planck constant, and $v$ is frequency of excited light. It is predicted that the $E Q E$ of G/A/G NW PD with 2DEHT structure (50) is significantly higher than the one (5.1) of pure GaAs NW PD, further confirming the enhanced efficiency of carrier generation, separation and collection of the 2DEHT structure. 
(a)

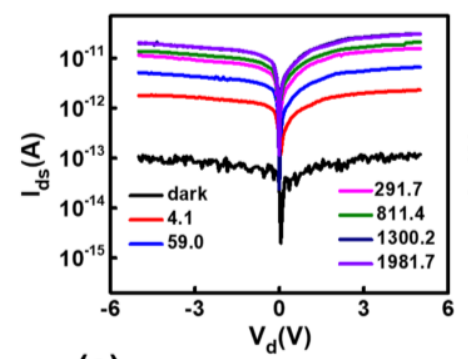

(c)

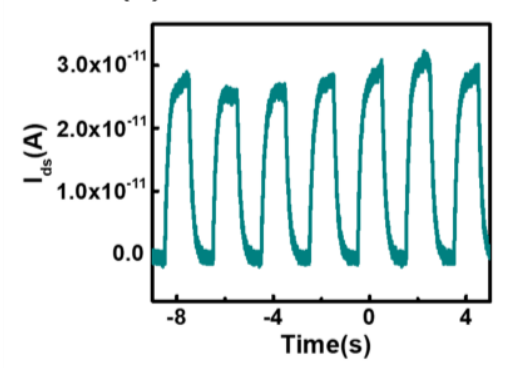

(b)

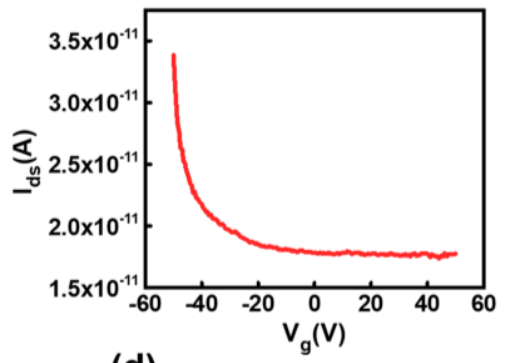

(d)

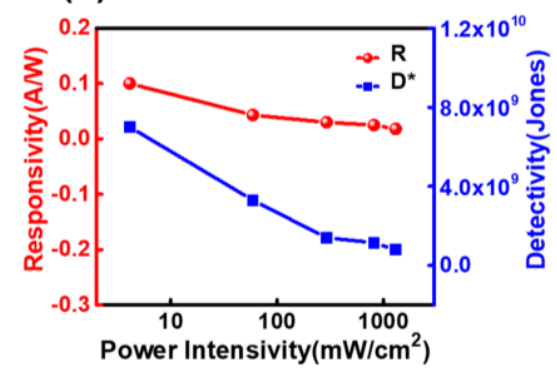

Figure S4. (a) Output characteristics of single unetched G/A/G NW photodetector under light illumination $(808 \mathrm{~nm}$ ) for different intensities at gate bias of $0 \mathrm{~V}$; (b) Transfer characteristics of the NW photodetector under forward bias sweep at room temperature; (c) Time-dependent photoresponse of the NW photodetector; (d) Responsivity and detectivity of the photodetector as a function of different illumination power intensity.

We assess the photoelectric characteristics of the photodetector fabricated with unetched G/A/G NW and evaluate its performance in Figure S4, in which this unetched G/A/G NW can be regarded as a regular GaAs NW with surface passivation for alleviated carrier recombination. Indeed, the responsivity of the unetched G/A/G device is nearly 2 times higher than that of the pure GaAs NW PD; however, this responsivity value is still obviously lower than that of the axially asymmetric G/A/G NW device. In explicit, Figure S4a shows the optical response characteristics of the device at gate voltage of $0 \mathrm{~V}$ and different illumination power densities. Figure S4b shows the device 
transfer characteristics under the forward bias sweeping condition at room temperature. It can be observed that the unetched NW also could yield the p-type semiconducting behavior. At the same time, the response time of the unetched G/A/G NW photodetector as shown in Figure S4c. It can be observed that its rise time and decay time are 199 and $240 \mathrm{~ms}$ respectively. The rise time of the unetched G/A/G NW photodetector is in between the rise time of the GaAs NW photodetector and the etched G/A/G NW photodetector. The rise time of the device is related to the carrier transmission process. Compared with the pure GaAs NW, the unetched G/A/G NW has a certain passivation effect, which can reduce the collision between the carriers and surface defects to some extent. For the etched G/A/G NW, it is obvious that the 2DEHT structure not only has a certain passivation effect, but also can dramatically reduce the collision between the carriers and the internal lattice defects. So, the rise time of unetched G/A/G NW PD is in between pure GaAs NW and etched G/A/G NW. The decay time is affected by the carrier recombination process in NW. Because there is no the 2DEHT structure formed in unetched $\mathrm{G} / \mathrm{A} / \mathrm{G} \mathrm{NW}$, the carriers are promiscuous distributed without sorted out and the recombination in the NW is similar to the pure GaAs NW, so the decay time is similar to the pure GaAs NW photodetector, slower than the etched G/A/G NW photodetector with 2DEHT. Notably, the parameters of $R$ and $D^{*}$ of the device under $-5 \mathrm{~V}$ bias are as well calculated in Figure $\mathrm{S} 4 \mathrm{~d} . R$ is evaluated as $0.11 \mathrm{~A} / \mathrm{W} ; D^{*}$ is 7.02 $\times 10^{9}$ Jones. It is evident that the unetched G/A/G NW photodetector does not perform as good as the axially asymmetric G/A/G NW photodetector. The reason is that the 2DEHT structure of the axially asymmetric G/A/G NW photodetector can effectively 
reduce the loss of the photogenerated carriers as compared with the surface passivated unetched G/A/G NW device. Also, the 2DEHT structure can efficiently separate the photogenerated carriers, which reduces the recombination of carriers, enhancing the photocurrent and thus having higher $R$ and $D^{*}$. 
(a)

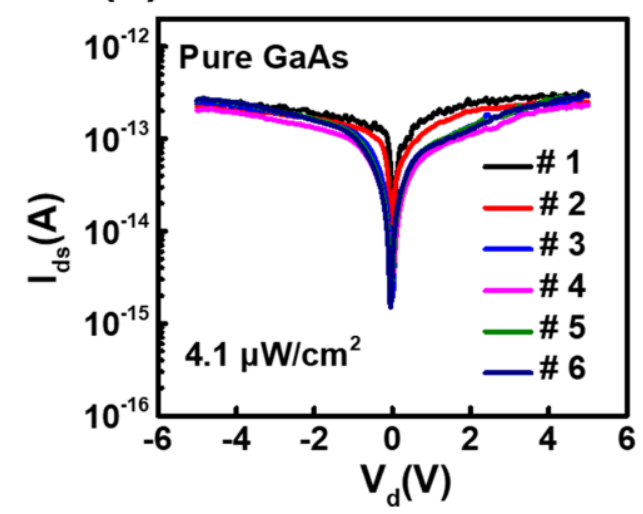

(b)

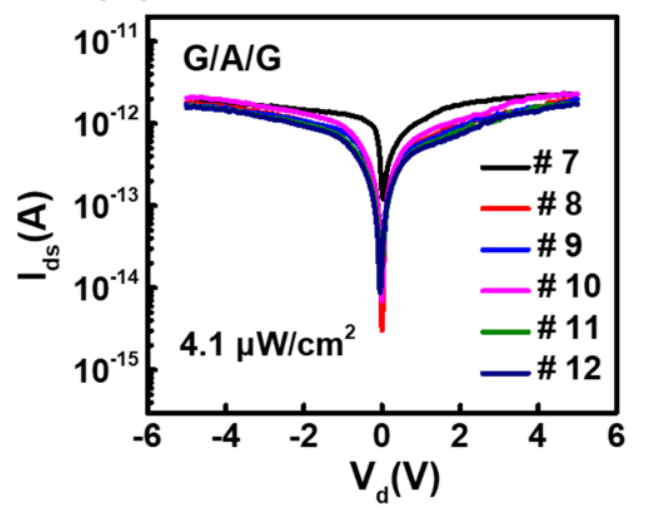

Figure S5. Output characteristics of photodetectors based on (a) a typical GaAs and (b) a G/A/G NW under light illumination of $808 \mathrm{~nm}$ for $4.1 \mu \mathrm{W} / \mathrm{cm}^{2}$ intensities.

\begin{tabular}{rll}
\hline & R(A/W) & D*(Jones) \\
\hline GaAs $\# 1$ & 0.078 & $3.02 \times 10^{9}$ \\
$\# 2$ & 0.061 & $2.28 \times 10^{9}$ \\
$\# 3^{*}$ & 0.073 & $2.69 \times 10^{9}$ \\
$\# 4$ & 0.056 & $1.99 \times 10^{9}$ \\
$\# 5$ & 0.071 & $2.75 \times 10^{9}$ \\
$\# 6$ & 0.070 & $2.50 \times 10^{9}$ \\
Average & 0.068 & $2.54 \times 10^{9}$ \\
\hline G/A/G \#7 & 0.62 & $2.01 \times 10^{10}$ \\
$\# 8$ & 0.50 & $1.57 \times 10^{10}$ \\
$\# 9^{*}$ & 0.57 & $1.83 \times 10^{10}$ \\
$\# 10$ & 0.49 & $1.61 \times 10^{10}$ \\
$\# 11$ & 0.45 & $1.44 \times 10^{10}$ \\
$\# 12$ & 0.43 & $1.40 \times 10^{10}$ \\
Average & 0.51 & $1.64 \times 10^{10}$ \\
\hline
\end{tabular}

Table S1. R and D* of GaAs NW photodetectors and axial asymmetry G/A/G NW photodetectors. The asterisk indicates the data selected in the manuscript.

Figure S5 shows the output characteristics of 6 groups of GaAs NW photodetectors and G/A/G NW photodetectors with 2DEHT structure. The device exhibits good reproducibility. It can be seen from Figure S5a that the photocurrents of GaAs NW photodetectors are in the range of $3.19 \times 10^{-13} \mathrm{~A}$ to $2.29 \times 10^{-13} \mathrm{~A}$ under $5 \mathrm{~V}$ bias and 
$4.1 \mu \mathrm{W} / \mathrm{cm}^{2}$ excitation power; the photocurrents of the $\mathrm{G} / \mathrm{A} / \mathrm{G} \mathrm{NW}$ photodetectors are in the range of $2.48 \times 10^{-12} \mathrm{~A}$ to $1.71 \times 10^{-12} \mathrm{~A}$ under the same conditions, as shown in Figure S5b. The R and D* of 6 groups of devices are listed in Table 1, and then the average values of performance are calculated. The average $\mathrm{R}$ of the GaAs $\mathrm{NW}$ photodetectors is $0.068 \mathrm{~A} / \mathrm{W}$, and the average $\mathrm{D}^{*}$ is $2.54 \times 10^{9}$ Jones. The average $\mathrm{R}$ of $\mathrm{G} / \mathrm{A} / \mathrm{G}$ NW photodetectors is $0.51 \mathrm{~A} / \mathrm{W}$, and the average $\mathrm{D}^{*}$ is $1.64 \times$ $10^{10}$ Jones. The average $\mathrm{R}$ of the device is increased by 7.5 times, and the average $\mathrm{D}^{*}$ is increased by 6.5 times. 
(a)

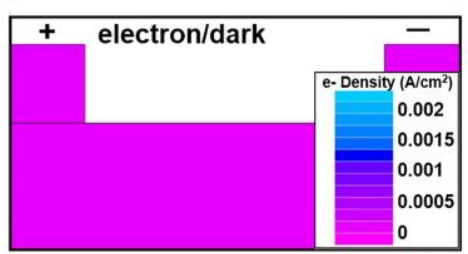

(c)

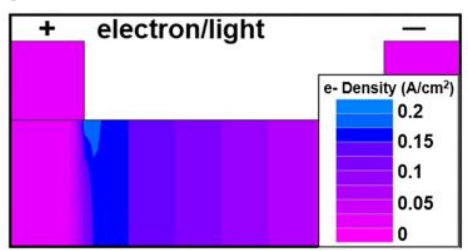

(b)

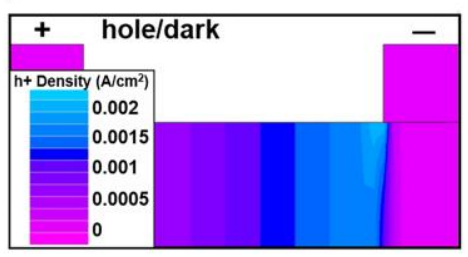

(d)

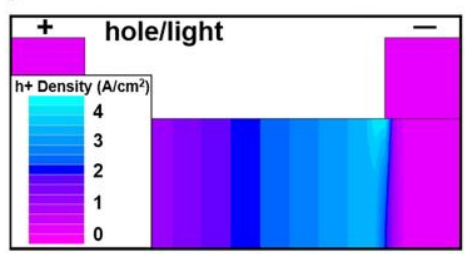

Figure S6. Two-dimensional profile of carrier distribution in GaAs NW photodetectors.

The simulated NW have a length of $4 \mu \mathrm{m}$ and a diameter of $200 \mathrm{~nm}$; (a) and (b) are the electron current density and hole current density maps of photodetectors in dark; (c) and (d) are the electron current density and hole current density maps of GaAs NW photodetector under $808 \mathrm{~nm}$ illumination at $-5 \mathrm{~V}$ bias.

For a consistent comparison, we also simulated the electron and hole concentration distributions of the pure GaAs NW under dark and illuminated conditions as shown in Figure S6. As discussed in the main text, the carrier concentration is generally low under dark. Because GaAs NWs are p-type here, the electron concentration can hardly be observed. Under $-5 \mathrm{~V}$ bias, the holes are transported to the right electrode and get collected as shown in Figures S6a, b. Under illumination, the electrons are collected by the left electrode and the holes are collected by the right electrode for the bias of $-5 \mathrm{~V}$ as shown in Figure S6c, d. It can be seen that both electron and hole concentrations of the GaAs photodetector are almost the same in the radial direction. They gradually change in the axial direction and reach the maximum concentration near the electrodes. 
(a)

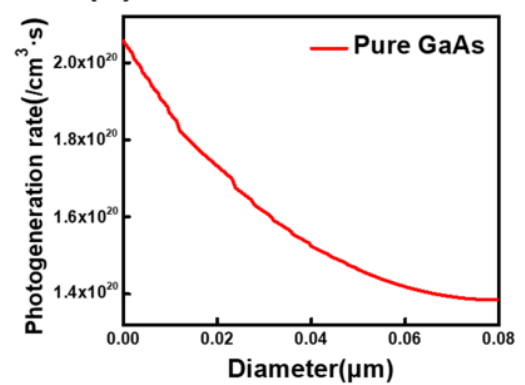

(c)

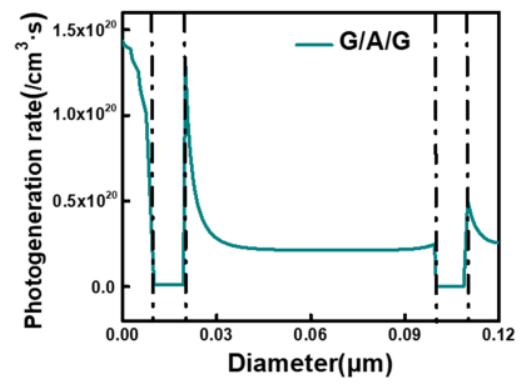

(b)

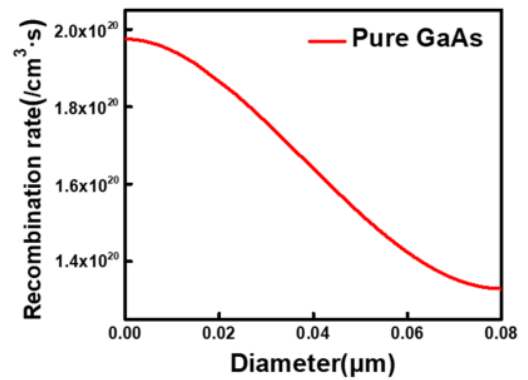

(d)

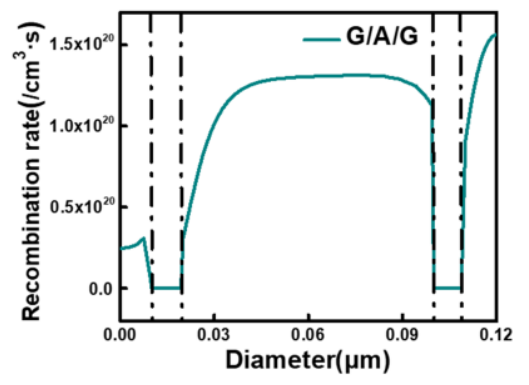

Figure S7. (a) Carrier concentration and (b) carrier recombination rate of simulated pure GaAs NW photodetector; (c) Carrier concentration and (d) carrier recombination rate of simulated axially asymmetric G/A/G NW photodetector.

Along the NW axial direction, carrier concentration distribution and carrier recombination rate of the pure GaAs NW and the axially asymmetric G/A/G NW are simulated by Silvaco Atlas as presented in Figure S7. Figure S7a shows the carrier concentration distribution of the GaAs NW (120 nm in diameter) under $808 \mathrm{~nm}$ illumination. Because the electrodes are located above the simulated device structure, the carrier concentration will gradually decrease when the axial carrier concentration is selected (shown from the left to the right). For the pure GaAs NW, the recombination rate of the part with the larger carrier concentration is also higher as shown in Figure S7b. Figure S7c shows the lateral structure of the NW $(10 \mathrm{~nm} \mathrm{GaAs} / 10 \mathrm{~nm}$ AlGaAs/ $/ 80$ $\mathrm{nm} \mathrm{GaAs} / 10 \mathrm{~nm} \mathrm{AlGaAs} / 10 \mathrm{~nm}$ GaAs) from the left to the right. The $808 \mathrm{~nm}$ 
illumination light source is excited from the left side. Since the NW studied here is a ptype semiconductor, once the photogenerated holes are generated by the GaAs NW core, they are substantially concentrated at the contact region between GaAs and AlGaAs. This way, the hole distribution in the inner layer is less, effectively separating holes and electrons, and thus reducing the probability of carrier recombination. As a result, the two-dimensional tube structure formed by GaAs and AlGaAs barriers can effectively bind the photogenerated holes, which can greatly reduce the recombination rate for the NW core as displayed in Figure S7d.

\section{Calculations}

\section{Responsivity and Detectivity}

In fact, responsivity $(R)$ is an important parameter of evaluating the performance of photodetectors. $^{2}$ It describes the photocurrent produced when the light illumination irradiates the effective part of NW device channel. It can be analytically expressed as follow:

$$
R=\frac{I_{\text {light }}-I_{\text {dark }}}{A * P}
$$

where $I_{\text {dark }}$ is the current obtained in the dark, $I_{\text {light }}$ is the current obtained under illumination, $A$ is the effective NW area, and $P$ is the light illumination power intensity. Another important figure-of-merit characterizing the photodetector is the detectivity $\left(D^{*}\right)$ as measurements shown in Figure $3 b . D^{*}$ assesses the sensitivity of device to the smallest detectable signal. ${ }^{3}$ It can be mathematically described as follow:

$$
D^{*}=\frac{R}{\left(2 e \cdot I_{\text {dark }}\right)^{1 / 2}}=\frac{I_{\text {light }}-I_{\text {dark }}}{A \cdot P\left(2 e \cdot I_{\text {dark }}\right)^{1 / 2}}
$$


where $R$ is the responsivity of device, $e$ is the electron charge, and $A$ is the effective NW area.

\section{Carrier Concentration and Recombination Rate}

To further shed light on the carrier recombination rate, we calculate the overall carrier concentration of the devices by measuring the actually obtained photocurrent, and then evaluated their carrier recombination rate using the carrier continuity equation. The relationship between photocurrent and carrier concentration can be mathematically expressed as follow:

$$
I_{\text {light }}=\left[\left(n_{0}+\Delta n\right)+\left(p_{0}+\Delta p\right)\right] e \cdot \mu \cdot \varepsilon \cdot s
$$

where $I_{\text {light }}$ is the photocurrent, $n_{0}$ is the electron concentration in equilibrium state, $p_{0}$ is the hole concentration in equilibrium state, $\Delta n$ is the photogenerated electron concentration, $\Delta p$ is the photogenerated hole concentration, $e$ is the electron charge, $\mu$ is the carrier mobility in non-equilibrium state, $\varepsilon$ is the electric field, and $s$ is the contact area between nanowires and electrodes. It is obvious that $\Delta n$ is equal to $\Delta p$. In this case, the hole concentration of the pure GaAs NW photodetector and the axially asymmetric G/A/G NW photodetector are determined to be $2.21 \times 10^{13}$ and $9.33 \times 10^{13}$ $/ \mathrm{cm}^{3}$, respectively. On the other hand, the relationship between carrier recombination rate and carrier concentration can be deduced by describing the carrier motion in nonequilibrium state through the carrier continuity equation and its boundary conditions as follows:

$$
\Delta \mathrm{p}=\tau_{p} g_{p}\left[1-\frac{s_{p} \tau_{p}}{L_{p}+s_{p} \tau_{p}} \exp \left(-\frac{r}{L_{p}}\right)\right]
$$

where $s_{p}$ is the rate of hole recombination, $\tau_{p}$ is the lifetime of the hole, $g_{p}$ is the 
rate of photogenerated hole, $r$ is the diffusion depth, and $L_{p}$ is the hole migration length. Because the light illumination can excite the entire NW, the actual diffusion depth $(r)$ becomes the diameter of NW. This way, the recombination rates are calculated to be $1.87 \times 10^{13}$ and $1.23 \times 10^{13} / \mathrm{cm}^{3} \cdot \mathrm{s}$ for the pure GaAs NW photodetector and the axially asymmetric $\mathrm{G} / \mathrm{A} / \mathrm{G} \mathrm{NW}$ photodetector, respectively.

\section{REFERENCES}

1. Hu, L.; Yan, J.; Liao, M.; Wu, L.; Fang, X. Ultrahigh External Quantum Efficiency from Thin $\mathrm{SnO}_{2}$ Nanowire Ultraviolet Photodetectors. Small 2011, 7, 1012 1017.

2. Ni, S.; Guo, F.; Wang, D.; Liu, G.; Xu, Z.; Kong, L.; Wang, J.; Jiao, S.; Zhang, Y.; Yu, Q.; Luo, J.; Wang, B.; Li, Z.; Zhang, C.; Zhao, L. Effect of MgO Surface Modification on the $\mathrm{TiO}_{2}$ Nanowires Electrode for Self-Powered UV Photodetectors. ACS Sustainable Chem. Eng. 2018, 6, 7265-7272.

3. Zou, R.; Zhang, Z.; Hu, J.; Sang, L.; Koide, Y.; Liao, M. High-detectivity nanowire photodetectors governed by bulk photocurrent dynamics with thermally stable carbide contacts. Nanotechnology 2013, 24, 495701. 